\title{
On the source-frequency dependence of fracture-orientation estimates from shear- wave transmission experiments
}

\author{
Léo Kirchhof Santos* - Universidade Federal do Pará, Faculdade de Geofísica \\ José Jadsom Sampaio de Figueiredo - Universidade Federal do Pará e Instituto nacional de Geofísica de petróleo (INCT \\ - GP), Brazil. \\ Bode Omoboya - Allied Geophysical Laboratories, University of Houston, Houston (TX), USA. \\ Jörg Schleicher - National Institute for Petroleum Geophysics (INCT-GP), Department of Applied Mathematics (DMA), \\ Institute of Mathematics, Statistics, and Scientific Computing (IMECC), University of Campinas (Unicamp) \\ Robert R. Stewart - Allied Geophysical Laboratories, University of Houston, Houston (TX), USA. \\ Nikolay Dyaur - Allied Geophysical Laboratories, University of Houston, Houston (TX), USA.
}

Copyright 2014, SBGf - Sociedade Brasileira de Geofísica

Este texto foi preparado para a apresentação no VI Simpósio Brasileiro de Geofísica, Porto Alegre, 14 a 16 de outubro de 2014. Seu conteúdo foi revisado pelo Comitê Técnico do VI SimBGf, mas não necessariamente representa a opinião da SBGf ou de seus associados. É proibida a reprodução total ou parcial deste material para propósitos comerciais sem prévia autorização da SBGt.

\section{Abstract}

Shear-wave propagation through anisotropic fractured or cracked media can provide valuable information about these fracture swarms and their orientations. The main goal of this work is to recover information about fracture orientation based on the shear waveforms (Swaveforms). For this study, we carried out ultrasonic Swave measurements in a synthetic physical model made of epoxy resin (isotropic matrix proxy), with small rubber strips as inclusions (artificial cracks) inserted in it to simulate a homogeneous anisotropic medium. In these experiments, we used low, intermediate, and high frequency shear-wave sources, with frequencies 90, 431 , and $840 \mathrm{kHz}$. We integrated and interpreted the resulting S-wave seismograms, cross-correlation panels and anisotropic parameter-analysis curves. We were able to estimate the crack orientation in singleorientation fracture zones. We applied a bandpass filtering process to the intermediate and high frequencies seismograms in order to obtain low frequency seismograms. A spectral analysis using frequency-wavenumber (F-K) spectra supports this filtering process. The results obtained using an analysis of cross-correlograms and the Thomsen parameter extracted from filtered high-frequency data were quite similar to those obtained using a low-frequency source. This highlighted the possibility of using less expensive high-frequency sources to recover information about the fracture set.

\section{Introduction}

In most reservoirs, the oil and gas production is controlled by fractures in the subsurface. In such reservoirs, the location, orientation, and density of fractures are important parameters to be characterized in order to optimize the production of fluids.

The Earth's subsurface has a very complex distribution of geological features such as fractures, faults and folds. Their characterization is very important for exploration geophysics, in especial for the oil and gas production. Among these features, the fractures have a very important paper, since they are, in most reservoirs, conducts for these fluids. Thus, their location, orientation, and density are important parameters to be characterized.
Some fractured reservoirs (particularly shale and carbonates) usually have high secondary porosity, however their permeability is usually low (Lonergan 2007; Nelson 2001). This permeability can be increased by hydraulic fracturing and knowing the preferential orientation of the fractures before this process is important to enhance the fluid flow (Holditch et al. 1978).

Due to the importance of fracture characterization, several methods have been developed in order to obtain information about fracture parameters.

De Figueiredo et al. (2012) used an analysis of lowfrequency $P$ and S-wave seismograms, crosscorrelation panels, and curves of Thomsen's anisotropy parameter (Thomsen 1986) to estimate the preferential fracture-set orientation of each region. This work complements their research on estimating fracture orientations. While de Figueiredo et al. (2012) used only low-frequency sources to obtain information about preferential fracture orientation in the model, we demonstrate the use of three different sources in the low (LF), intermediate (IF), and high-frequency (HF) domain, with 90,431 and $840 \mathrm{kHz}$, respectively.

We use a bandpass filter to obtain low-frequency information. Before the filtering process, the seismograms are converted into F-K spectra in order to visualize the signals' frequency distribution. The analysis of de Figueiredo et al. (2012) efficiently extracted fracture orientation information when the $S$ wave propagation was normal to the bedding planes. The main step in this work is the interpretation of seismograms, high coherence values of correlograms and anisotropy parameter curves for both original and filtered datasets. Similar to de Figueiredo et al. (2012), the interpretation considers the fracture orientation as an unknown parameter. However, as a main assumption to the interpretation, other fracture parameters, such as the size of the inclusions and the crack density, are considered known.

\section{Methodology}

We studied the effect of oriented fractures on elastic wave propagation in a synthetic epoxy-resin model with embedded neoprene-rubber cracks. The construction of the anisotropic cracked sample as well as the ultrasonic measurements was carried out at the Allied Geophysical Laboratories (AGL) at the University of Houston, Texas. The experimental setup used to make the samples and carry out the S-waveform measurements was the same as used in Omoboya et al. (2011), de Figueiredo et al. (2012), Stewart et al. (2013), and de Figueiredo et al. (2013). It is described 
in detail in these works. Therefore, we restrict ourselves For the physical-model experiments, we constructed two epoxy samples. The first sample, denominated $M$, was constructed layer by layer, interrupted by the introduction of rubber cracks. After addition of each 5 $\mathrm{mm}$ thick epoxy layer with solid neoprene-rubber inclusions to the sample, we extracted the air using a vacuum pump to avoid air bubbles in the epoxy resin. It consists of three regions with neoprene inclusions simulating different crack-set orientations and crack densities. The other sample, $R$, is an isotropic reference model without inclusions, produced in a single cast. On sample $M$, we used five different measurement positions, labeled $\mathrm{M}-1$ to $\mathrm{M}-5$ in Figure 1a. Positions $\mathrm{M}-$ $1, M-3$, and $M-5$ are located in the center of the three differently fractured regions, while $\mathrm{M}-2$ and $\mathrm{M}-4$ are at the boundaries between them. We determined a ratio between the compressional wave velocities in solid epoxy resin and neoprene rubber of approximately 1:5. The S-wave velocity in the neoprene rubber was difficult to determine because of the low shear modulus of this material. The wavelengths for body waves propagating in the model were in the range of $1.9-2.5 \mathrm{~cm}$ for $P$ waves and $1.0-1.4 \mathrm{~cm}$ for $S$ waves.

The geometrical parameters of the included rubber-strip cracks in the cracked model are displayed in Table 1, where we estimated the crack density $\epsilon_{c}$ in each region of sample M according to the Hudson (1981) formula,

$$
\epsilon_{c}=\frac{N V_{c}}{V}=\frac{N l h^{2}}{V}
$$

Here, $N$ is the number of cracks, $V_{c}$ is the volume of a single crack, and $\mathrm{V}$ is the volume of the model. For our strip-shaped cracks, $V_{c}=I h 2$, where $\mathrm{I}$ is the crack length and $h$ is the crack aperture.

The Ultrasonic Research System at AGL, relies on Swave transducers with central frequencies at $90 \mathrm{kHz}$, $431 \mathrm{kHz}$ and $840 \mathrm{kHz}$, respectively, which can be rotated to allow for different S-wave polarizations. In the experiments used in this work, we started with polarization parallel to the $X$ axis and then rotated the source and receiver transducers 18 times, by $10^{\circ}$ at a time (i.e., $0^{\circ}$ to $180^{\circ}$ ), until the polarization was again in the original $X$ direction. We repeated this process for the cases of S-wave propagation in the $\mathrm{Y}$ and $\mathrm{Z}$ directions (see Figure 1b). We acquired each of the 19 output traces with a 20 -fold stack to eliminate ambient noise. For the velocity computations, we picked arrival times at the first wave maxima, subtracting a delay of $2.7 \mu$ s from the observed arrival time. The time-picking accuracy was 0:2 $\mu \mathrm{s}$, which yields an error in the estimated velocities of about $4 \mathrm{~m} / \mathrm{s}$.

Table 1- Geometrical parameters of the reference model $(R)$ and the three regions $(M$ 1, M-3, M5) of the cracked model. Source: Figueiredo, Schleicher e Stewart (2012)] here to a brief outline of the physical experiments.

\begin{tabular}{lccccccc}
\hline \hline Model & $\begin{array}{c}\text { Crack } \\
\text { density (\%) }\end{array}$ & \multicolumn{2}{c}{$\begin{array}{c}\text { Measuring } \\
\text { length model }(\mathrm{cm}) \\
L_{Z}\end{array}$} & $\begin{array}{c}\text { Number } \\
\text { of layers }\end{array}$ & $\begin{array}{c}\text { Cracks } \\
\text { per layer }\end{array}$ & $\begin{array}{c}\text { Crack } \\
\text { length }(\mathrm{cm})\end{array}$ & $\begin{array}{c}\text { Crack } \\
\text { aper.(cm) }\end{array}$ \\
\hline R & Isotropic & 7.51 & 7.62 & 0 & 0 & - & - \\
M-1 & 4.5 & 7.56 & 7.89 & 10 & 36 & 0.8 & 0.2 \\
M-3 & 4.5 & 7.56 & 7.89 & 10 & 36 & 0.8 & 0.2 \\
M-5 & 4.5 & 7.59 & 7.81 & 10 & 36 & 0.8 & 0.2
\end{tabular}

(a)

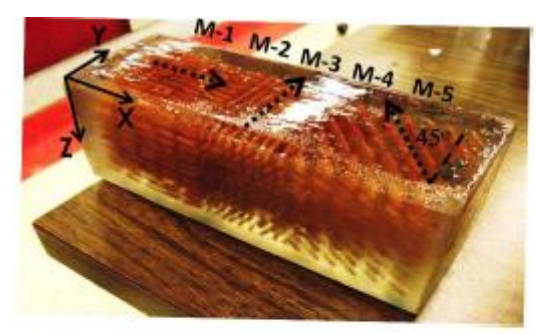

(b)

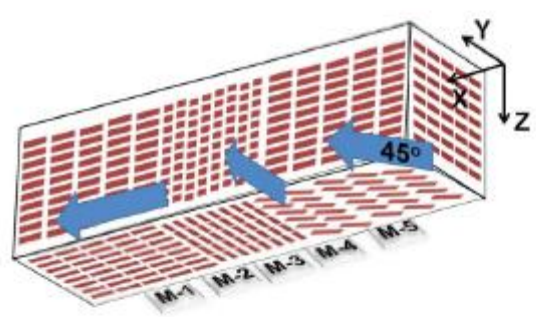

(c)

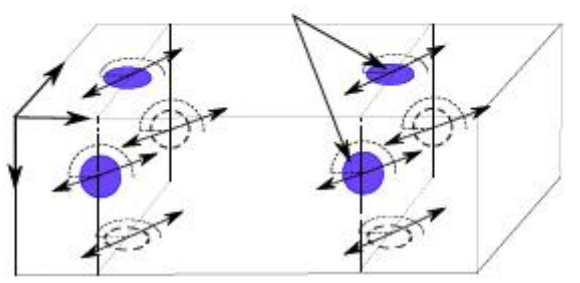

Figure 1 - (a) Photograph of the fractured model showing three regions with the same crack density but with three different set orientations. (b) Schematic diagram of crack placement of $Y X$ (bedding plane) and $Z X$ planes of the fracture model. (c) Schematic representation of the $S$-wave transducer placing at the centers of opposite sides of the model. Source: Figueiredo, Schleicher $e$ Stewart (2012).

\section{Theory}

The analyses are based on the two S-wave phases, being the fast S-wave (S1) and slow S-wave (S2). These phases are generated when the S-wave enters an anisotropic medium, in this case a synthetic cracked sample, and splits into these two phases. In a fractured medium, the faster S1 wave is always polarized parallel to the fractures, while the slower S2 wave is always polarized perpendicular to them. 
Besides analyzing the S-wave seismograms, we also investigate two seismic attributes: correlograms and Thomsen's parameter curves.

According to Yilmaz (2001), the similarity between two different traces in time can be measured by means of a cross-correlation analysis. Kennett (2002) observes that cross-correlation of S-wave phases $\mathrm{S} 1(\mathrm{t})$ and $\mathrm{S} 2(\mathrm{t})$ can be employed to estimate S-wave polarization, $\theta$, and time delay, $\Delta \mathrm{t}$, between these two phases. This information is important because shear-wave polarization is related to crack orientation (Crampin 1985) while the time delay is related to crack distribution (Hudson 1981).

For this reason, we cross-correlated the waveforms of the S-wave seismograms. The basic idea of the polarization measurement by cross-correlation relies on two recordings, $S(\varphi ; t)$ and $H(\varphi ; t)$, with polarizations orthogonal to each other. These recordings are assumed to contain two orthogonal shear waves ( $\mathrm{S} 1$ (t) and $\mathrm{S} 2(\mathrm{t})$ ) with the same time function, but separated in time by a delay $\Delta t$. Angle $\varphi$ denotes the (unknown) deviation between the trial polarizations of $S(\varphi ; t)$ and $\mathrm{H}(\varphi ; t)$ from the true polarizations of $\mathrm{S} 1(\mathrm{t})$ and $\mathrm{S} 2(\mathrm{t})$. In terms of the two fundamental shear waves $S 1(t)$ and $S 2(t)$, the traces $S(\varphi ; t)$ and $H(\varphi ; t)$ can be represented by (Kennett 2002)

$$
\begin{aligned}
S(\phi, t) & =S 1(t) \cos (\phi)+S 2(t+\delta t) \sin (\phi), \\
H(\phi, t) & =S 1(t) \sin (\phi)-S 2(t+\delta t) \cos (\phi) .
\end{aligned}
$$

According to its definition (see, e.g., Yilmaz 2001), the cross-correlation function between the two traces represented by Equations (2) can be written as

$$
R(\phi, \delta t)=\sum_{i=1}^{n} S\left(\phi_{1}, t_{i}\right) H\left(\phi, t_{i}+\delta t\right)
$$

where $\mathrm{n}$ is the window length (time window) of the operation (the size of the trace). The correlograms depicted below are generated by correlating the first trace $(\varphi 1=0)$ with each of the 19 traces.

It is well established that Thomsen parameter describes the influence of the medium anisotropy on S-wave propagation (Thomsen 1986). This parameter is related to the velocities of the orthogonal shear-waves polarizations, S1 and S2. In this work, we are using the same notation for as described in Thomsen (1986)

$$
\gamma=\frac{C_{66}-C_{44}}{2 C_{44}}=\frac{1}{2}\left(\frac{V_{S 1}^{2}}{V_{S 2}^{2}}-1\right),
$$

where $\mathrm{C}_{66}=\rho \mathrm{V}^{2}{ }_{\mathrm{S} 1}$ and $\mathrm{C} 44=\rho \mathrm{V}^{2} \mathrm{~s} 2$ are elastic stiffness coefficients, $\rho$ is the medium density, and $V_{s 1}$ and $V_{s 2}$ are the velocities of the S1 and S2 waves.

The density is absent in Equation (4) because the density in our physical model experiments is always constant.

Rewriting Equation (4) as function of S1 and S2 traveltimes $\mathrm{t} S 1$ and $\mathrm{t} S 2$, we obtain

$$
\gamma=\frac{1}{2}\left(\frac{\left(\Delta L / t_{S 1}\right)^{2}}{\left(\Delta L / t_{S 2}\right)^{2}}-1\right)=\frac{1}{2}\left(\frac{t_{S 2}^{2}}{t_{S 1}^{2}}-1\right),
$$

where $\Delta \mathrm{L}$ is the distance traveled by the S-waves from the source to the receiver positions. The fact that this distance is the same for S1 and S2 polarizations allows to simplify Equation (5) to arrive at the right-hand expression.

Equation 5 shows that Thomsen parameter is directly related to the ratio between $t S 2$ and $t S 1$, which are the highest and lowest S-wave traveltimes observed in a fractured medium. In this work, we used the traveltimes for different polarization angles in order to determine the preferential fracture orientation in a region independently of the above correlograms. For this purpose, we extracted the traveltimes automatically from the central lobes of the recorded wavelet. We then estimate the value of

$$
\Gamma(\theta)=\frac{1}{2}\left(\frac{t\left(\theta+90^{\circ}\right)^{2}}{t(\theta)^{2}}-1\right)
$$

for all polarization angles $\theta$ ranging from $0^{\circ}$ to $90^{\circ}$. The highest value of this ratio is expected to occur when the polarization angle $\theta$ is perpendicular to the dominant fracture orientation, i.e., when the polarization $\theta+90^{\circ}$ is parallel to the fracture orientation.

Figure 6 depicts the $\Gamma$ curves extracted from the unfiltered seismograms for propagation both in the $Z$ and $Y$ directions. As expected, the $\Gamma$ curves in the reference medium (top row) are at, indicating that the S-wave propagation is isotropic. At the 5 measurement points in the fractured sample, the maximum values in the LF $\Gamma$ curves indeed occur at the expected polarization angles.

The IF $\Gamma$ curves show a similar behavior, but with smaller amplitudes, making them less reliable to interpret. The HF $\Gamma$ curves, on the other hand, are practically constant at all measurement points, exhibiting a behavior similar to the reference curve. This situation occurs because the wavelength of these high-frequency waves is very small, smaller than the fractures. As a consequence, these waves are insensitive to the anisotropy caused by the fractures. It is not possible to conclude anything about fracture orientation using these high-frequency y curves.

The same procedure applied to the low-pass filtered seismograms demonstrates that $\Gamma$ curves can be used to reliably estimate fracture orientations even from higher-frequency seismograms. We see in Figure 7 that after filtering, the IF and $\mathrm{HF} \Gamma$ curves exhibit approximately the same behavior as the LF $\Gamma$ curves. For instance, at each of the three frequencies, we can observe that the largest value of $\Gamma$ in region $M-1$ occurs when the angle of rotation is $0^{\circ}$ and the largest value of $\Gamma$ in region M-3 occurs when the polarization is at an angle of $90^{\circ}$.

From these observations, we can conclude that the fracture set orientation in regions $\mathrm{M}-1$ and $\mathrm{M}-3$ are parallel to the $X$ and $Y$ directions, respectively. The rather low amplitudes at measurement points $M-2, M-4$, and $M-5$ indicate that the information extracted from these $\Gamma$ curves might be faulty. 


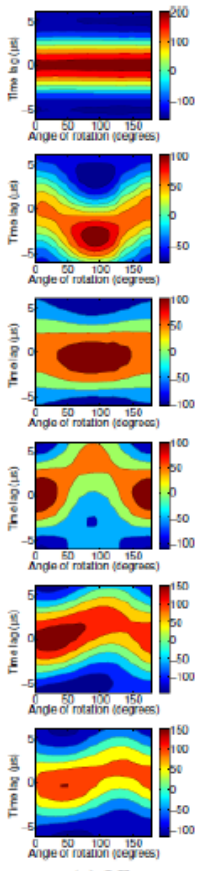

(a) LF
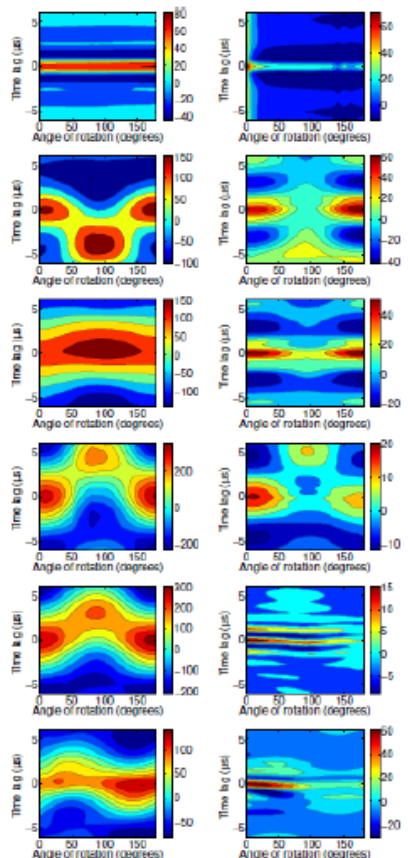

(b) IF
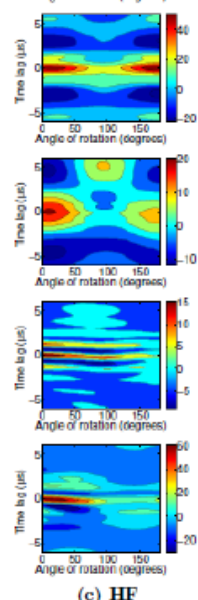

Figure 2 - $S$-wave correlograms related to $L F$ and unfiltered seismograms for propagation in $Z$ direction. The Figures depicted in the first row corresponds to the reference $(R)$ sample and the other rows in descending order are the correlograms for positions $M-1$, $M-2, M-3, M-4$, and $M-5$
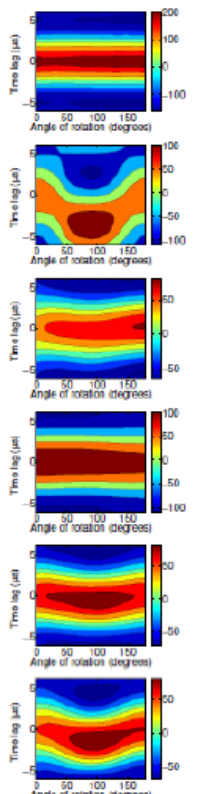

(a) $\mathbf{L F}$
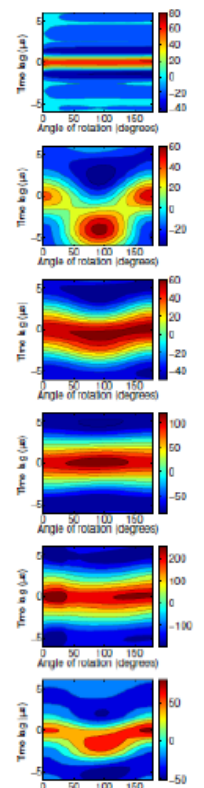

(b) IF
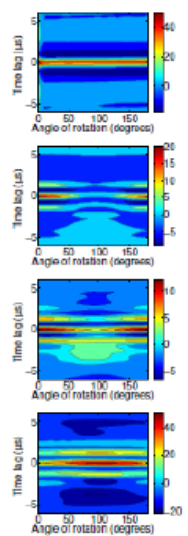

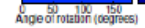

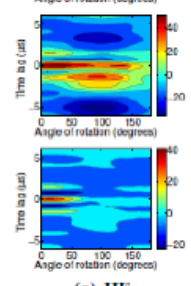

(c) $\mathrm{HF}$

Figure 3 - S-wave correlograms related to $L F$ and unfiltered seismograms for propagation in $Y$ direction. The Figures depicted in the first row corresponds to the reference $(R)$

sample and the other rows in descending order are the correlograms for positions $M-1$, $M-2, M-3, M-4$, and $M-5$

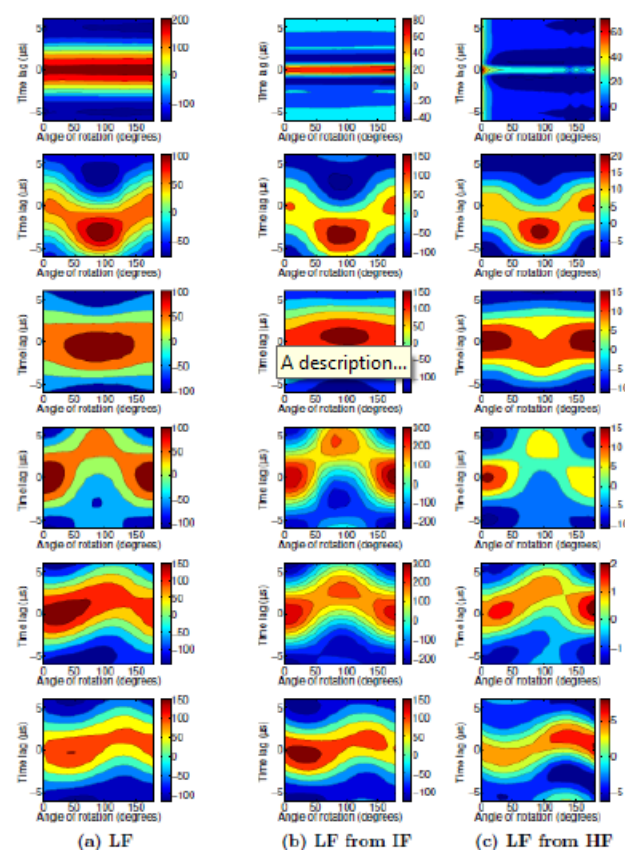

Figure 4 - $S$-wave correlograms related to $L F$ and filtered seismograms for propagation in $Z$ direction. The Figures depicted in the first row corresponds to the reference $(R)$ sample and the other rows in descending order are the correlograms for positions $M-1, M-2, M$ 3, $M-4$, and $M-5$

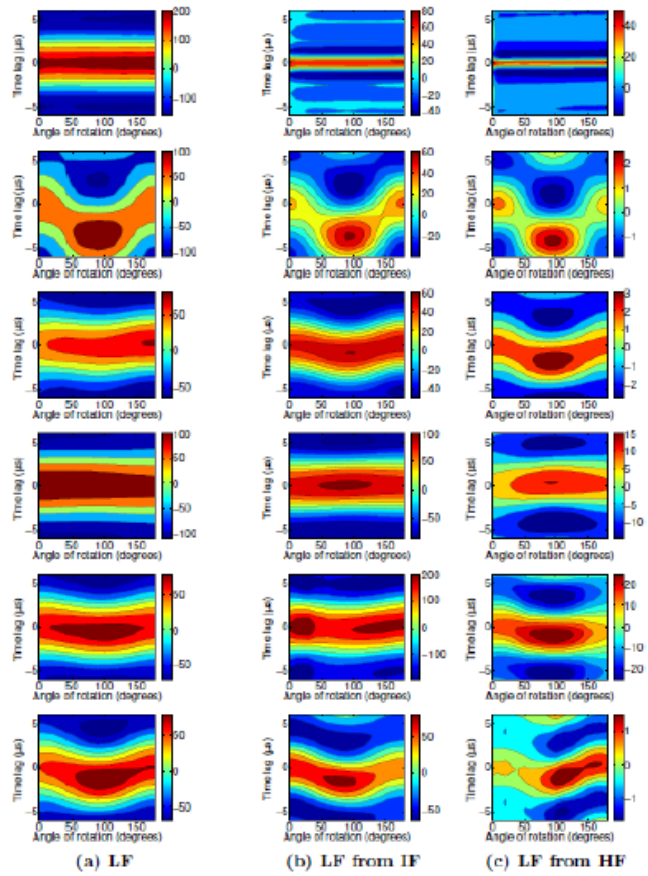


Figure 5 - $S$-wave correlograms related to $L F$ and filtered seismograms for propagation in $Y$ direction. The Figures depicted in the first row corresponds to the reference $(R)$ sample and the other rows in descending order are the correlograms for positions $M-1, M-2, M$ 3, $M-4$, and $M-5$
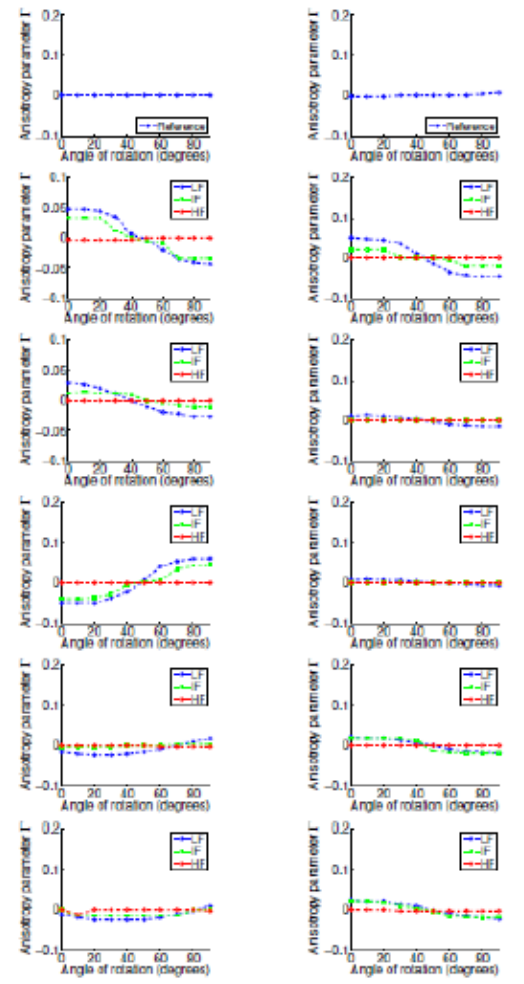

(a) $\mathrm{Z}$ direction (b) $\mathrm{Y}$ direction

Figure 6 - Anisotropy parameter curves for low frequency and intermediate and high frequencies unfiltered. Figures depicted in the first row corresponds to the reference $(R)$ sample and other lines in descending order correspond to curves for positions $M-1, M-2$, $M-3, \quad M-4$, and $M-5$. First column corresponds to the curves associated to $S$ wave propagation in $Z$ direction while the second column corresponds to $S$-wave propagation in $Y$ direction
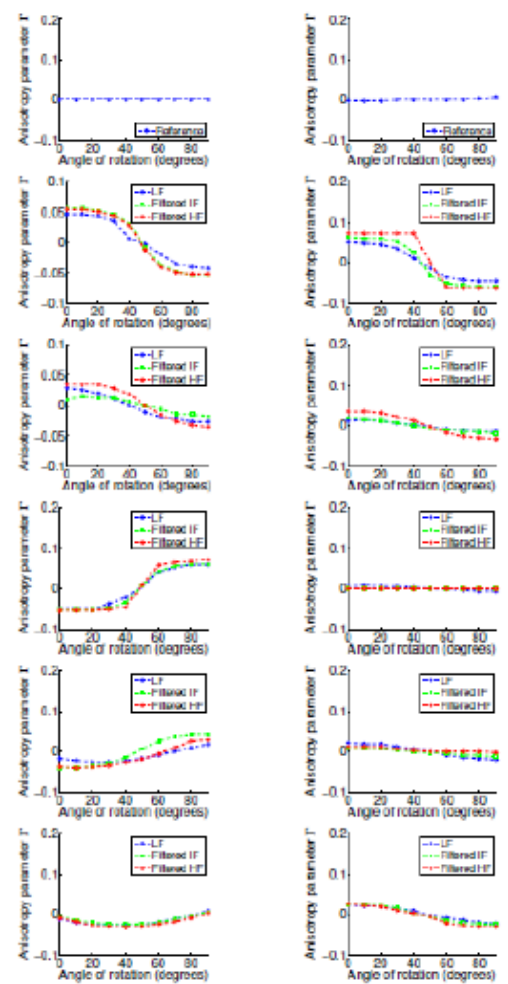

(a) $\mathrm{Z}$ direction

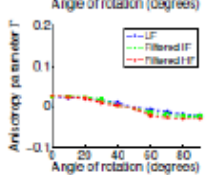

(b) $\mathbf{Y}$ direction

Figure 7 - Anisotropy parameter curves for original low frequency and intermediate and high frequencies filtered. Figures depicted in the first row corresponds to the reference $(R)$ sample and other lines in descending order correspond to curves for positions $M-1, M-2$, M-3, M-4, and M-5. First column corresponds to the curves associated to $S$ wave propagation in $Z$ direction while the second column corresponds to $S$-wave propagation in $Y$ direction

\section{Discussion and conclusions}

In this work, we have extended the analysis of de Figueiredo et al. (2012) in order to estimate the fracture orientation. We used the analysis of S-wave velocity and frequency in order to recover information about the predominant orientation of the fracture sets in a physical model with differently cracked regions. From the seismograms, we generated cross-correlation panels and anisotropy parameter curves, which helped us in achieving our goal. Integrating the results of these techniques, we were able to demonstrate their capability of estimating some characteristics of the model.

This work showed that low-frequency seismic data is the most important source of information on fracture orientation, but high-frequency sources can provide additional information from both filtered and unfiltered signals. Moreover, wave propagation parallel to the bedding planes and parallel to the axis of the cracks (as in the region at $\mathrm{M}-3$ in our model for propagation in the $Y$ direction), causes almost isotropic behavior in the 
curves and panels. In such situations, noise in the data might lead, in all implemented techniques, to low amplitude oscillations prone to incorrect interpretation. Additional information can help to confirm quasiisotropic behavior. All techniques tested in this work are immediately applicable in cross-well studies.

\section{References}

Assad, J. M., R. H. Tatham, and J. A. McDonald, de 1992, A physical model study of microcrackinduced anisotropy, Geophysics, 57 (12), 1562\{1570, doi: 10.1190/1.1443224.

Crampin, S., de 1985, Evaluation of anisotropy by shearwave splitting, Geophysics, 50 (1), 142\{152, doi:10.1190/1.1441824.

de Figueiredo, J. J. S., J. Schleicher, R. R. Stewart, and N. Dyaur, de 2012, Estimating fracture orientation from elastic-wave propagation: An ultrasonic experimental approach, Journal of Geophysical Research, 117 (B8), doi:10.1029/2012JB009215.

de Figueiredo, J. J. S. d., J. Schleicher, R. R. Stewart, N. Dayur, B. Omoboya, R. Wiley, and A. William, de 2013, Shear wave anisotropy from aligned inclusions: ultrasonic frequency dependence of velocity and attenuation, Geophysical Journal International, 193 (1), 475\{488, doi:10.1093/gji/ggs130.

Far, M. E., C. M. Sayers, L. Thomsen, D.-h. Han, and J. P. Castagna, de 2013, Seismic characterization of naturally fractured reservoirs using amplitude versus o_set and azimuth analysis, Geophysical Prospecting, 61 (2), 427447, doi:10.1111/1365-2478.12011.

Holditch, S., J. Jennings, S. Neuse, and R.Wyman (1978), The optimization of well spacing and fracture length in low permeability gas reservoirs, Society of Petroleum Engineers, doi:10.2118/7496-MS.

Hudson, J. A., de 1981,Wave speeds and attenuation of elastic waves in material containing cracks, Geophysical Journal of the Royal Astronomical Society, $64 \quad(1), \quad 133\{150, \quad$ doi:10.1111/j.136546X.1981.tb02662.x.

Kennett, B. L. N., de 2002, The Seismic Wave_eld: Volume 2, Interpretation of Seismograms on Regional and Global Scales, Cambridge University Press.

Li, R., P. Okoye, and N. Uren, de 2000, A study of the effects of transducer size on physical modeling experiments for recovering anisotropic elastic parameters, Geophysical Research Letters, 27 (22), 36433646, doi: 10.1029/2000GL011401.

Lonergan, L., de 2007, Fractured Reservoirs, Geological Society of London.

Nelson, R., de 2001, Geologic Analysis of Naturally Fractured Reservoirs, Gulf Professional Publishing.
Omoboya, B., J. J. S. de Figueiredo, N. Dyaur, and R. R. Stewart, de 2011, Uniaxial stress and ultrasonic anisotropy in a layered orthorhombic medium, pp. 2145\{2149, Society of Exploration Geophysicists, doi:10.1190/1.3627634.

Quan, Y., and J. M. Harris, de 1997, Seismic attenuation tomography using the frequency shift method, Geophysics, 62 (3), 895\{905, doi:10.1190/1.1444197.

Sacchi, M. D., D. J. Verschuur, and P. M. Zwartjes (2004), Data reconstruction by generalized deconvolution, pp. 1989\{1992, Society of Exploration 25 Geophysicists, doi:10.1190/1.1843303.

Stewart, R. R., N. Dyaur, B. Omoboya, J. J. S. de Figueiredo, M. Willis, and S. Sil, de 2013, Physical modeling of anisotropic domains: Ultrasonic imaging of laser-etched fractures in glass, Geophysics, 78 (1), D11\{D19, doi: 10.1190/geo2012-0075.1.

Thomsen, L, de. 1986, Weak elastic anisotropy, Geophysics, $\quad 51 \quad$ (10), $1954\{1966$, doi:10.1190/1.1442051

Tillotson, P., J. Sothcott, A. I. Best, M. Chapman, and X.-Y. Li (2012), Experimental verification of the fracture density and shear-wave splitting relationship using synthetic silica cemented sandstones with a controlled fracture geometry, Geophysical Prospecting, 60 (3), 516\{525, doi:10.1111/j.13652478.2011.01021.x.

Willis, M. E., D. R. Burns, R. V. N. Rao, B. J. Minsley, M. N. Toksoz, and L. Vetri, de 2005, Spatial orientation and distribution of reservoir fractures from scattered seismic energy, Technical report, Massachusetts Institute of Technology. Earth Resources Laboratory, shortened title: Fracture characterization from coda waves.

Yilmaz, O., de 2001, Seismic Data Analysis: Processing, Inversion, and Interpretation of Seismic Data, SEG Books.

Zhang, Y., X. Campman, S. K. Grandi, S. Chi, M. N. Toksoz, M. E. Willis,

D. R. Burns, and L. Vetri, de 2006, F-k characteristics of the seismic response to a set of discrete parallel fractures, Technical report, Massachusetts Institute of Technology. Earth Resources Laboratory, united States. Dept. of Energy (award number DE-FC2602NT15346). 